\title{
Structure-property relationships in hybrid dental nanocomposite resins containing monofunctional and multifunctional polyhedral oligomeric silsesquioxanes
}

\author{
This article was published in the following Dove Press journal: \\ International Journal of Nanomedicine \\ 10 February 2014 \\ Number of times this article has been viewed
}

\author{
Weiguo Wangl,** \\ Xiang Sun ${ }^{1, *}$ \\ Li Huang 2,* \\ Yu Gao' \\ Jinghao Ban' \\ Lijuan Shen' \\ Jihua Chen' \\ 'Department of Prosthodontics, \\ 2Department of General Dentistry \\ and Emergency, School of \\ Stomatology, Fourth Military Medical \\ University, Xi'an, People's Republic \\ of China \\ *These authors contributed equally to \\ this work
}

Correspondence: Jihua Chen

Department of Prosthodontics, School of Stomatology, Fourth Military Medical University, Changle West Road I45,

Xi'an, 710032 People's Republic of China

Tel +86298477 6329

Fax +86298477 6329

Email jhchen@fmmu.edu.cn

\begin{abstract}
Organic-inorganic hybrid materials, such as polyhedral oligomeric silsesquioxanes (POSS), have the potential to improve the mechanical properties of the methacrylate-based composites and resins used in dentistry. In this article, nanocomposites of methacryl isobutyl POSS (MI-POSS [bears only one methacrylate functional group]) and methacryl POSS (MA-POSS [bears eight methacrylate functional groups]) were investigated to determine the effect of structures on the properties of dental resin. The structures of the POSS-containing networks were determined by scanning electron microscopy, transmission electron microscopy, X-ray diffraction, and Fourier transform infrared spectroscopy. Monofunctional POSS showed a strong tendency toward aggregation and crystallization, while multifunctional POSS showed higher miscibility with the dimethacrylate monomer. The mechanical properties and wear resistance decreased with increasing amounts of MI-POSS, indicating that the MI-POSS agglomerates act as the mechanical weak point in the dental resins. The addition of small amounts of MAPOSS improved the mechanical and shrinkage properties. However, samples with a higher MA-POSS concentration showed lower flexural strength and flexural modulus, indicating that there is a limited range in which the reinforcement properties of MA-POSS can operate. This concentration dependence is attributed to phase separation at higher concentrations of POSS, which affects the structural integrity, and thus, the mechanical and shrinkage properties of the dental resin. Our results show that resin with 3\% MA-POSS is a potential candidate for resinbased dental materials.
\end{abstract}

Keywords: POSS, methacrylate monomer, dental resin, hybrid material, nanocomposite

\section{Introduction}

Dental composite resins have been widely used in restorative treatments since their introduction about 50 years ago. The resin matrix usually consists of several methacrylate monomers and is cured by photoinitiated free radical polymerization. Compared to dental amalgams, they have fewer safety concerns and better esthetic properties. ${ }^{1,2}$ However, the composite resins in current use still suffer from two key shortcomings: high polymerization shrinkage and low mechanical properties. ${ }^{2-4}$ Considerable efforts have been devoted to the improvement of the mechanical and shrinkage properties by optimization of inorganic filler type and content, with some success in improving the immediate mechanical properties. ${ }^{1,3,5}$ However, many studies also reported that failure still occurred when the composite materials were subjected to prolonged use; the median survival lifespan of resin-based composites is about 5 to 7 years, much 
shorter than dental amalgam (13 years)..$^{1,3,6}$ The major reason proposed for this failure is the bond between the fillers and the resin, provided by silane coupling agents, which can be degraded by water absorbed by the composites after longterm use. ${ }^{5}$ Thus, there remains a pressing need to improve the performance of dental composite resins.

Organic-inorganic materials are considered a new generation of high-performance materials, as they combine the advantages of inorganic materials (eg, rigidity and high stability) with those of organic polymers (eg, flexibility, ductility, and processability). Polyhedral oligomeric silsesquioxanes (POSS) are one of the typical organic-inorganic hybrid nanomaterials, which were developed toward the end of the last century. POSS are $1-3 \mathrm{~nm}$ in diameter and have a compact hybrid structure, with an inorganic core made up of silicon and oxygen $\left(\mathrm{SiO}_{1.5}\right)_{\mathrm{n}}$ (Figure 1). POSS can be thought of as analogs of the smallest possible silica particles. ${ }^{7,8}$ However, unlike silicas, silicones, or other nanofillers, each POSS molecule contains either unreactive organic functional groups, which help POSS molecules become soluble in, and compatible with, polymers or reactive organic functional groups, making POSS molecules more suitable for polymerization or grafting. A previous study found that the incorporation of POSS into polymers resulted in improved biocompatibility and reduced inflammatory response, ${ }^{9}$ which make this material suitable for oral use.

Depending on its functionality, POSS can be incorporated as a pendant unit in the network if it bears only one reactive group (monofunctional POSS), or POSS can be incorporated as a junction (cross-linking point) if it bears more than two reactive groups (multifunctional POSS). ${ }^{10}$ Multifunctional POSS can be used as cross-linkers for polymers or as the main com-

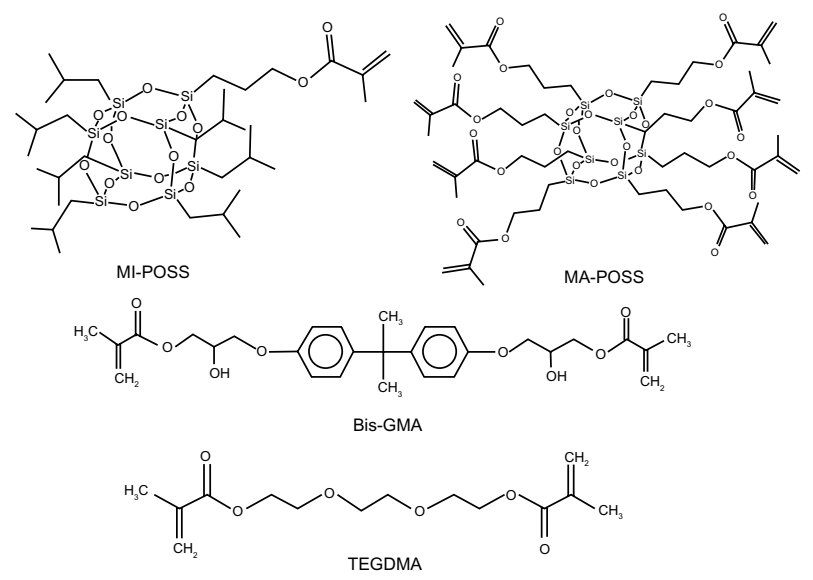

Figure I Chemical structures of the two types of POSS and the resin monomers. Abbreviations: Bis-GMA, bisphenol A glycerolate dimethacrylate; MA-POSS, methacryl polyhedral oligomeric silsesquioxanes; MI-POSS, methacryl isobutyl polyhedral oligomeric silsesquioxanes; POSS, polyhedral oligomeric silsesquioxanes; TEGDMA, tri(ethylene glycol) dimethacrylate. ponent in the elaboration of hybrid materials. Monofunctional POSS, meanwhile, can serve as anchoring points bound to the matrix chains. These characteristics make POSS molecules attractive candidates for a wide range of applications. Both monofunctional and multifunctional POSS, with suitable reactive functional groups, have been used as modifiers and cross-linkers for polystyrene, epoxy, polycarbonate, and other polymer matrices. The resulting copolymers and networks usually display improved viscoelastic and mechanical properties, high thermal stability and oxygen permeability, and low thermal expansion. ${ }^{11-14}$ However, only a few studies have addressed dental composite resins, ${ }^{15-17}$ and any differences between monofunctional and multifunctional POSS in dental composites have not been reported.

The aim of this research was to determine how different functionalized POSS structures of the organic substituent on the inorganic cage would affect the compatibility, photocuring behaviors, morphologies, mechanical properties, and shrinkage properties of methacrylate-based dental composite resins. The hypothesis was that uniform distribution of well-separated POSS into bisphenol A glycerolate dimethacrylate (Bis-GMA) and tri(ethylene glycol) dimethacrylate (TEGDMA) dental composite resins would result in substantial improvements in these properties.

\section{Materials and methods Materials}

The chemical structures of the different reactants are shown in Figure 1. Methacryl isobutyl POSS (MI-POSS) and methacryl POSS (MA-POSS) were purchased from Hybrid Plastics Company (Hattiesburg, MS, USA). Ethyl 4-dimethylaminobenzoate (EDMAB) and camphorquinone (CQ) were purchased from Sigma-Aldrich Company (St Louis, MO, USA). Bis-GMA, TEGDMA, and the $0.7 \mu \mathrm{m}$ silinated barium borosilicate glass (V-117-4107) were provided by Esstech, Inc (Essington, PA, USA). All materials were used as received without further purification.

\section{Dental composite preparation}

The organic matrix contained Bis-GMA and diluent comonomer TEGDMA (Figure 1). CQ and EDMAB were added as the initiator and coinitiator, respectively. Two types of POSS, MA-POSS and MI-POSS, were used as reinforcing materials. They were first dispersed in TEGDMA, followed by mechanical stirring for 2 hours at $400 \mathrm{rpm}$. Bis-GMA, CQ, and EDMAB were then added, and the system was mechanically stirred for another 2 hours at $400 \mathrm{rpm}$. The formulations of the resin systems investigated in this study are described in Table 1 . The 
effects of different concentrations of POSS on the dental resins were studied, and the two types of POSS were incorporated into the resin matrices at concentrations of $1,3,5$, and $10 \mathrm{wt} \%$. The composites with 1, 3, 5, and $10 \mathrm{wt} \%$ of MA-POSS were abbreviated as MA1, MA3, MA5, and MA10, respectively; and composites with $1,3,5$, and $10 \mathrm{wt} \%$ of MI-POSS were abbreviated as MI1, MI3, MI5, and MI10, respectively.

All materials were mixed sufficiently in the dark. To eliminate the influence of the barium glass filler on the microstructure, the experiments involving scanning electron microscopy (SEM), wide-angle X-ray diffraction (WAXD), and degree of double bond conversion (DC) used net resin without filler. For shrinkage, wear, and mechanical test specimens, a mass fraction of $70 \%$ of silinized fine-milled barium glass powder (Esstech Inc, Essington, PA, USA) was added. In this study, all specimens were photocured with the same light curing unit, $600 \mathrm{~mW} / \mathrm{cm}^{2}$ (Spectrum ${ }^{\mathrm{TM}} 800$; DENTSPLY, York, PA, USA). For the neat resin systems without filler, specimens were photocured for 20 seconds, while the resin systems with filler were photocured for 60 seconds on each side. The process of adding the filler into the dental resin resulted in decreased curing depth. Thus, for the resin with filler, we increased the irradiation time to combat the effects of decreased curing depth.

\section{Morphology}

The morphology of the fractured surface was examined using a field emission scanning electron microscope

Table I The code and compositions of dental resin systems

\begin{tabular}{ll}
\hline Code & $\begin{array}{l}\text { Compositions of dental resin system } \\
(x=1,3,5,10) \text { wt\% }\end{array}$ \\
\hline Control & Bis-GMA/TEGDMA/CQ/EDMAB =49.5/49.5/0.5/0.5/ \\
MAI & Bis-GMA/TEGDMA/CQ/EDMAB/MA-POSS =49/49/0.5/0.5/I \\
MA3 & Bis-GMA/TEGDMA/CQ/EDMAB/MA-POSS $=48 / 48 / 0.5 / 0.5 / 3$ \\
MA5 & Bis-GMA/TEGDMA/CQ/EDMAB/MA-POSS =47/47/0.5/0.5/5 \\
MAI0 & Bis-GMA/TEGDMA/CQ/EDMAB/MA-POSS =44.5/44.5/0.5/0.5/I0 \\
MII & Bis-GMA/TEGDMA/CQ/EDMAB/MI-POSS $=49 / 49 / 0.5 / 0.5 / 1$ \\
MI3 & Bis-GMA/TEGDMA/CQ/EDMAB/MI-POSS $=48 / 48 / 0.5 / 0.5 / 3$ \\
MI5 & Bis-GMA/TEGDMA/CQ/EDMAB/MI-POSS $=47 / 47 / 0.5 / 0.5 / 5$ \\
MII0 & Bis-GMA/TEGDMA/CQ/EDMAB/MI-POSS $=44.5 / 44.5 / 0.5 / 0.5 / / 0$
\end{tabular}

Abbreviations: Bis-GMA, bisphenol A glycerolate dimethacrylate; $\mathrm{CQ}$, camphorquinone; EDMAB, ethyl 4-dimethylamino-benzoate; MAI, resin with I wt\% of methacryl polyhedral oligomeric silsesquioxanes group; MA3, resin with 3 wt\% of methacryl polyhedral oligomeric silsesquioxanes; MA5, resin with $5 \mathrm{wt} \%$ of methacryl polyhedral oligomeric silsesquioxanes; MAI0, resin with 10 wt\% of methacryl polyhedral oligomeric silsesquioxanes; MA-POSS, methacryl polyhedral oligomeric silsesquioxanes; MII, resin with I wt\% of methacryl isobutyl polyhedral oligomeric silsesquioxanes group; MI3, resin with $3 \mathrm{wt} \%$ of methacryl isobutyl polyhedral oligomeric silsesquioxanes; MI5, resin with $5 \mathrm{wt} \%$ of methacryl isobutyl polyhedral oligomeric silsesquioxanes; MII 0 , resin with $10 \mathrm{wt} \%$ of methacryl isobutyl polyhedral oligomeric silsesquioxanes; MI-POSS, methacryl isobuty polyhedral oligomeric silsesquioxanes;TEGDMA, tri(ethylene glycol) dimethacrylate.
(S-4800; Hitachi Ltd, Tokyo, Japan). The samples were made of neat resin without barium glass filler to eliminate the influence of barium glass filler on the microstructure. The specimens were stored in deionized water for 24 hours at $37^{\circ} \mathrm{C}$ in the dark and then broken off by hand. The photographs were then taken for the fractured surface. The samples' surfaces were coated with a thin layer of gold (E-1045; Hitachi Ltd) before testing because of the poor electrical conductivity of the samples. Energy-dispersive X-ray spectrometry (EDAX; Mahwah, NJ, USA) coupled with field emission-SEM showed the distribution of silicon atoms in the hybrid. A transmission electron microscope (TEM) (Tecnai G2; FEI, Hillsboro, OR, USA), at an accelerating voltage of $120 \mathrm{kV}$, was used to further investigate the dispersion of MA-POSS as well as the resulting microstructure of the dental matrix. The TEM samples were cut using an ultramicrotome to $\sim 70-90 \mathrm{~nm}$ thickness and mounted on carbon-coated copper TEM grids.

\section{Degree of conversion}

The degree of conversion of the unfilled resin systems was measured using a Fourier transform infrared (FTIR) spectrometer (8100 M; Shimadzu, Kyoto, Japan) at a resolution of $4 \mathrm{~cm}^{-1}$ and 32 scans in the range of $4,000-400 \mathrm{~cm}^{-1}$. A $20 \mu \mathrm{L}$ volume of the neat resin was applied to a potassium bromide $\operatorname{disc}(2.0 \mathrm{~cm} \times 2.0 \mathrm{~cm})$. Absorbance peaks before curing were recorded with the FTIR spectrometer. The unfilled resin systems were then photocured for 20 seconds with the light curing unit. FTIR examination was performed again at different time points (20,40, and 60 seconds and 2, 5, and 10 minutes) from the start of irradiation. Five specimens were tested for each group. The degree of DC (DC\%) was determined from the ratio of absorbance intensities of aliphatic $\mathrm{C}=\mathrm{C}$ (peak at $1,637 \mathrm{~cm}^{-1}$ ) against the internal reference aromatic $\mathrm{C}=\mathrm{C}$ (peak at 1,607 $\mathrm{cm}^{-1}$ ) before and after curing of the specimen. The degree of conversion was then calculated as,

$$
\begin{aligned}
\text { DC }(\%)= & \left(1-\frac{\left[1,637 \mathrm{~cm}^{-1} / 1,607 \mathrm{~cm}^{-1}\right] \text { peak area cured }}{\left[1,637 \mathrm{~cm}^{-1} / 1,607 \mathrm{~cm}^{-1}\right] \text { peak area uncured }}\right) \\
& \times 100
\end{aligned}
$$

\section{X-ray characterization}

WAXD of neat resins was conducted on the film samples, and MI-POSS samples were tested in powder form. The MA-POSS samples were not tested because MA-POSS is a liquid with an amorphous structure. The film specimens were manufactured between two glass slide coverslips with two coverslips sandwiched as thickness control. The specimens 
were photocured for 20 seconds and then carefully removed from the mold. Diffraction patterns were obtained using an X'Pert Pro MPD diffractometer (PANanlytical, Almelo, the Netherlands) in transmission mode at room temperature, using copper K-alpha radiation at a tube current of $44 \mathrm{~mA}$ and an acceleration voltage of $40 \mathrm{kV}$. Data points were collected over the $2 \theta$ range of $5^{\circ}-35^{\circ}$, at $0.02^{\circ}$ intervals.

\section{Volumetric shrinkage}

The volumetric shrinkage was determined by measuring the density $(\rho)$ of uncured and cured composite resin specimens, using a pycnometer (Elite Medical Co., Ltd, Nanjing, Jiangsu, People's Republic of China) according to the ISO 3521 standard. The photoactivation was conducted through the glass of the pycnometer, and all specimens were photocured for 60 seconds on each side. The volumetric shrinkage was determined 60 minutes after irradiation. The tests were done at $20^{\circ} \mathrm{C}-22^{\circ} \mathrm{C}$ and at a relative humidity of $50 \%$. The volumetric shrinkage $(\Delta \mathrm{V} / \mathrm{V}) \%$ was calculated using the formula,

$$
\left(\frac{\Delta \mathrm{V}}{\mathrm{V}}\right) \%=\frac{\rho_{\text {cured }}-\rho_{\text {uncured }}}{\rho_{\text {cured }}} \times 100 \%
$$

where $\rho_{\text {uncured }}$ is the density of specimen prior to curing, and $\rho_{\text {cured }}$ is the density of specimen after curing. Six specimens were tested for each group.

\section{Mechanical properties}

Flexural strength (FS) and flexural modulus (E) were measured in a three-point bending test. Specimens were manufactured in stainless steel molds $(2 \mathrm{~mm} \times 2 \mathrm{~mm} \times 25 \mathrm{~mm})$. To overcome the disadvantage of the long length of the specimens, we moved the light guide slowly along the specimens when photocuring. The specimens were photocured for 60 seconds on each side, and then carefully removed from the molds. All four sides of each specimen were carefully handpolished after 24 hours of storage in deionized water at $37^{\circ} \mathrm{C}$ in the dark. A universal testing machine (EZ-Test; Shimadzu) was used for the three-point bending test, according to the ISO 4049 standard, with a span of $20 \mathrm{~mm}$ and a crosshead speed of $0.5 \mathrm{~mm} /$ minute. Flexural strength and flexural modulus were calculated from the following formulas:

$$
\begin{gathered}
\mathrm{FS}=3 P L / 2 b h^{2} \\
E=\left(\frac{F}{d}\right)\left(\frac{L^{3}}{4 b h^{3}}\right)
\end{gathered}
$$

where $P$ is the load at fracture, $L$ is the distance between two supports (which was set at $20 \mathrm{~mm}$ ), $b$ is the width of the specimen, $h$ is the thickness of the specimen, $F$ is the $\operatorname{load}(\mathrm{N})$ at a point in the straight line portion of the trace, $d$ is the deflection (mm) at load $F$ and $F / d$ is the slope of the load-deflection curve. Six replicates were prepared for each sample.

\section{Wear resistance}

Disc-shaped specimens ( $8 \mathrm{~mm}$ diameter and $4 \mathrm{~mm}$ height) of each group were manufactured in stainless steel molds. The specimens were photocured for 60 seconds on each side, and then carefully removed from the molds and stored in deionized water at $37^{\circ} \mathrm{C}$ for 24 hours. The specimens were then positioned in the center of a silicone mold and the acrylic resin. The silicone molds had an internal diameter of $25.0 \pm 0.1 \mathrm{~mm}$ and were compatible with the chambers of the tribometer (CSM Instruments, Peseux, Switzerland). After 45 minutes, the mounted specimens were removed from the molds and carefully hand-polished. Six specimens were made for each group.

The tribometer was used to determine the wear behavior of the dental composite. Wear measurements were carried out at room temperature in deionized water with a normal load of $10 \mathrm{~N}$. The static partner was a stainless steel ball of $6 \mathrm{~mm}$ diameter cleaned with acetone. The specimens were subjected to 20,000 wear cycles before being removed from the chambers of the tribometer. The composite specimens were then scanned using the 3D Non-Contact profilometer (Nanovea Inc, Irvine, CA, USA). An area of $4 \mathrm{~mm}^{2}(2 \mathrm{~mm} \times 2 \mathrm{~mm})$ was scanned on the surface, coincident with the center of the worn area. The maximum wear depth ( $\mathrm{mm})$ of the wear facet was quantified using the nonworn areas as the reference points and used as indicator of the amount and degree of wear.

\section{Statistical analysis}

The acquired data of DC, volumetric shrinkage, FS, E, and maximum wear depth were analyzed with two-way analysis of variance (ANOVA) followed by post hoc Bonferroni analysis using IBM SPSS Statistics, version 20 (IBM Corporation, Armonk, NY, USA). References to significant differences were based on a probability of $P<0.05$. A normality test (Shapiro-Wilk) and an equal variance test for different results were performed before analysis of variance. All normality tests and equal variance tests were passed.

\section{Results and discussion Morphology of the hybrid materials}

The degree of dispersion of POSS moieties in polymer matrices has a great effect on both the thermal and mechanical properties of the resultant composites. ${ }^{18}$ For this reason, the fractured composite surfaces were studied by SEM and 
EDAX, with the aim of establishing the level of dispersion of POSS molecules in the matrix. Figure 2A shows low magnification SEM photomicrographs of fractured surfaces of MA10. The fracture surface was homogeneous, with no obvious phase separation or gaps. Figure 2B shows an EDAX silicon map of Figure 2A. The particles were uniformly dispersed throughout the polymer matrix. The micrographs for control, MA1, MA3, and MA5 are not shown because of their similarity to that shown in Figure 2A. Dental monomers do not contain any silicon, therefore, the silicon detected in the continuous matrix could only represent the molecular level of incorporation of POSS within the resin. Figure $2 \mathrm{C}$ and $\mathrm{E}$ show high magnification SEM pictures of fractured surfaces of MA5 and MA10, respectively. The fracture surface of MA5 was still homogeneous and the silicon particles in EDAX (Figure 2D) were uniformly dispersed throughout the polymer matrix. However, for MA10, several smooth clumps were observed in the matrix, and the EDAX figure in Figure 2F indicates the domains contained higher silicon
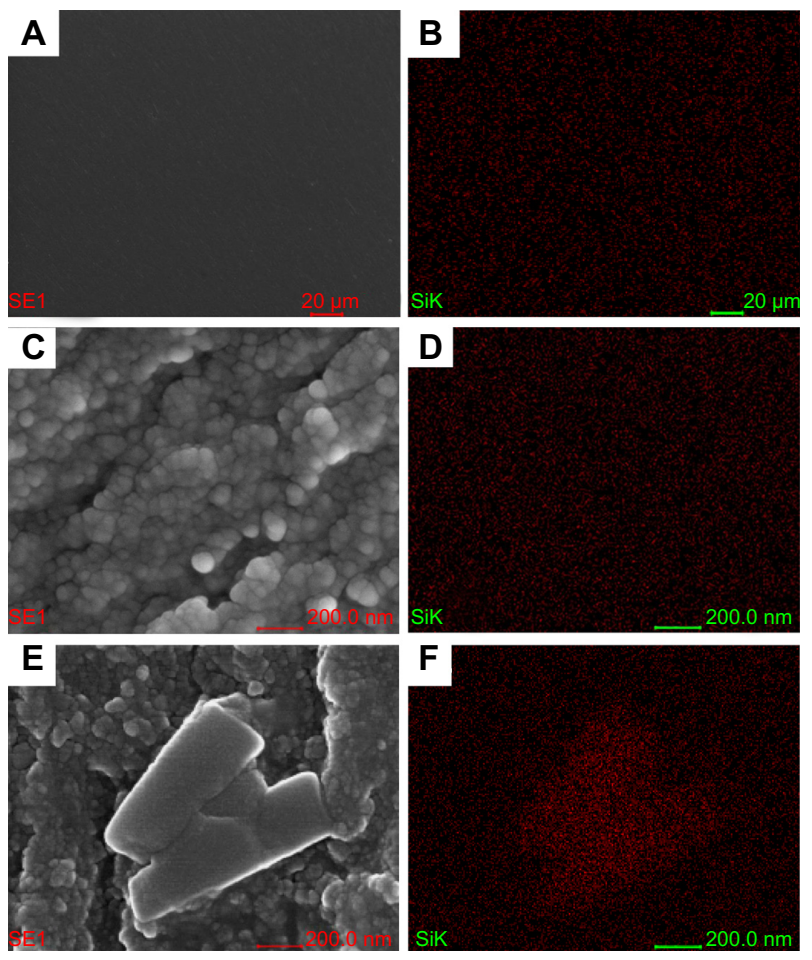

Figure 2 Scanning electron microscope and energy-dispersive $\mathrm{X}$-ray spectrometer micrographs of MA5 and MAI0.

Notes: (A) Low magnification scanning electron microscope micrograph of MAI0. (B) Low magnification energy-dispersive $X$-ray spectrometer micrograph of MAI0. (C) High magnification scanning electron microscope micrograph of MA5 (D) High magnification energy-dispersive X-ray spectrometer micrograph of MA5. (E) High magnification scanning electron microscope micrograph of MAIO. (F) High magnification energy-dispersive X-ray spectrometer micrograph of MAIO.

Abbreviations: MA5, resin with 5 wt\% of methacryl polyhedral oligomeric silsesquioxanes group; MAI0, resin with $10 \mathrm{wt} \%$ of methacryl polyhedral oligomeric silsesquioxanes group; SEI, electrons generated at the point of primary electron beam impingement in surface of the specimen; SiK, K shell of silicon electron shell. contents than the matrix, which could be attributed to the formation of POSS-enriched agglomerates.

Figure 3 shows the SEM-EDAX, micrographs of MIPOSS filled composites. As can be seen, a poor dispersion of the MI-POSS was detected, as shown by the accumulation of silicon signals indicating POSS aggregation. Moreover, the size and quantity of the agglomerates clearly increased with MI-POSS content. Monofunctional POSS has been found to cause similar agglomerates in several other receptors. ${ }^{13,19}$ The formation of the irregular agglomerates could be explained by POSS-POSS interaction and the mechanism of polymerization inducing phase separation. ${ }^{20}$ This phase separation occurred when mixing resin matrix and MI-POSS because of the incompatibility between the isobutyl groups of the POSS molecules and the aromatic groups of the Bis-GMA molecules. Additionally, even when reacted into the network,
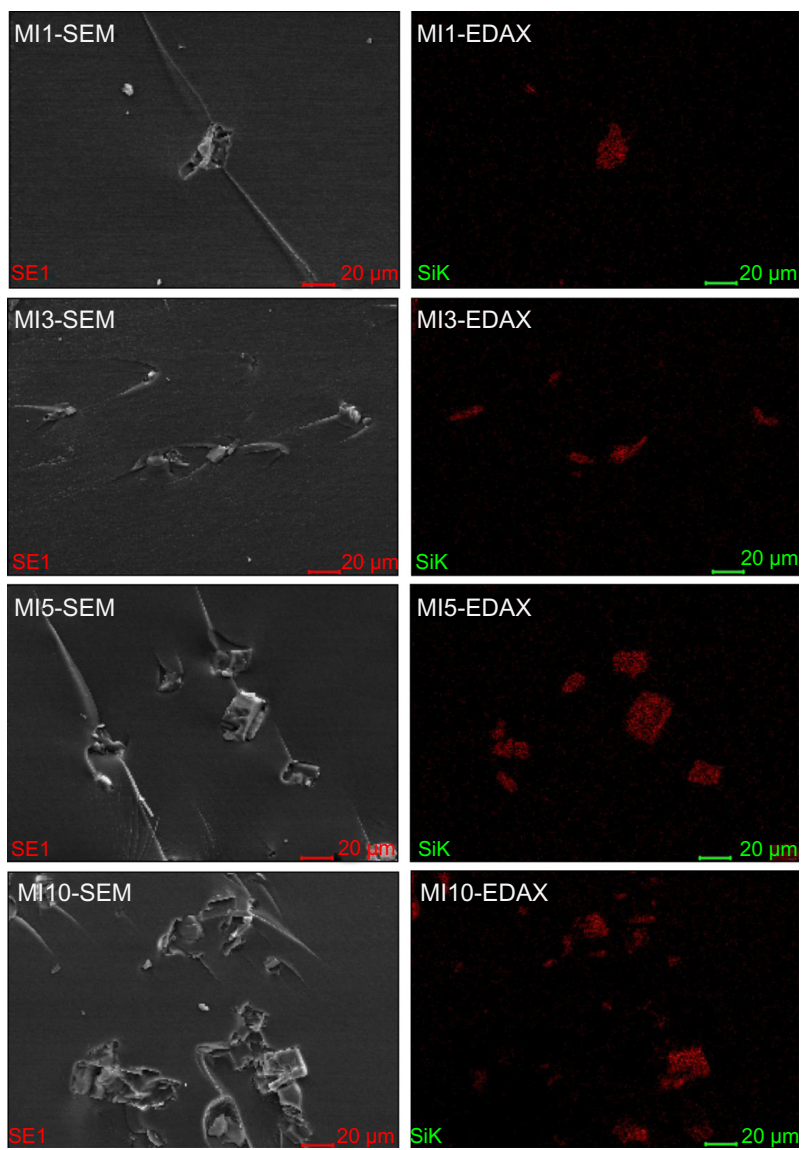

Figure 3 Low magnification scanning electron microscope (left panels) and energy-dispersive X-ray spectrometer micrographs (right panels) of resins filled with MI-POSS.

Abbreviations: EDAX, energy-dispersive X-ray; MI-POSS, methacryl isobutyl polyhedral oligomeric silsesquioxanes; MII, resin with I wt\% of methacryl isobutyl polyhedral oligomeric silsesquioxanes group; MI3, resin with $3 w t \%$ of methacryl isobutyl polyhedral oligomeric silsesquioxanes; MI5, resin with 5 wt\% of methacryl isobutyl polyhedral oligomeric silsesquioxanes; MIIO, resin with $10 \mathrm{wt} \%$ of methacryl isobutyl polyhedral oligomeric silsesquioxanes; SEM, scanning electron microscopy. 


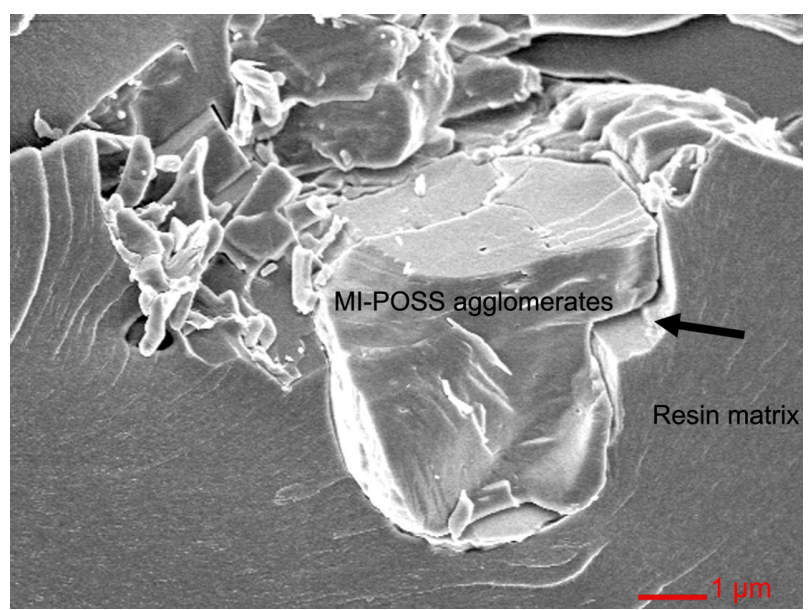

Figure 4 High magnification scanning electron microscope micrographs of MIIO. Note: Arrow shows the gap between agglomerate and resin matrix.

Abbreviations: MIIO, resin with $10 \mathrm{wt} \%$ of methacryl isobutyl polyhedral oligomeric silsesquioxanes group; MI-POSS, methacryl isobutyl polyhedral oligomeric silsesquioxanes.

the pendant POSS units are not completely constrained; ${ }^{12}$ POSS molecules with cage-like nanostructures tend to form random aggregations to reduce interfacial energy.

As can be seen from Figures 2E and 4, agglomerates of composites with $10 \mathrm{wt} \%$ MA-POSS have a different structure from agglomerates of composites with MI-POSS, which are shown in Figure 5. Although MA-POSS agglomerates were formed, they were still connected with the matrix, indicating that MA-POSS around the agglomerates was covalently bound to the molecular chains. Unlike MA-POSS agglomerates, there is a gap between MI-POSS agglomerates and matrix (Figure 4). This gap may suggest that they adhere to each other weakly. These MI-POSS agglomerates were significantly deleterious to the properties of composite resins, as will be will be shown later in this paper. In addition, MI-POSS agglomerates were much larger than MA-POSS agglomerates.

The MA-POSS-filled resin samples were selected for TEM measurements, and the results are shown in Figure 6. The TEM micrographs show dark zones that correspond to the POSS-rich zones (higher electron density because of the presence of silicon atoms). ${ }^{21}$ The resulting nanocomposites clearly show some agglomerates of the nanoparticles. The ubiquitous presence of these nanocrystallites indicates that, though there was good compatibility between MA-POSS and the dental matrix, some degree of MA-POSS aggregation still occurs at the nanoscale. The agglomerates resolved by TEM were significantly smaller than the agglomerates resolved by SEM. The TEM photomicrographs showing a cross section of aggregation may result in smaller agglomerates than SEM. SEM is very useful for observing three dimensional
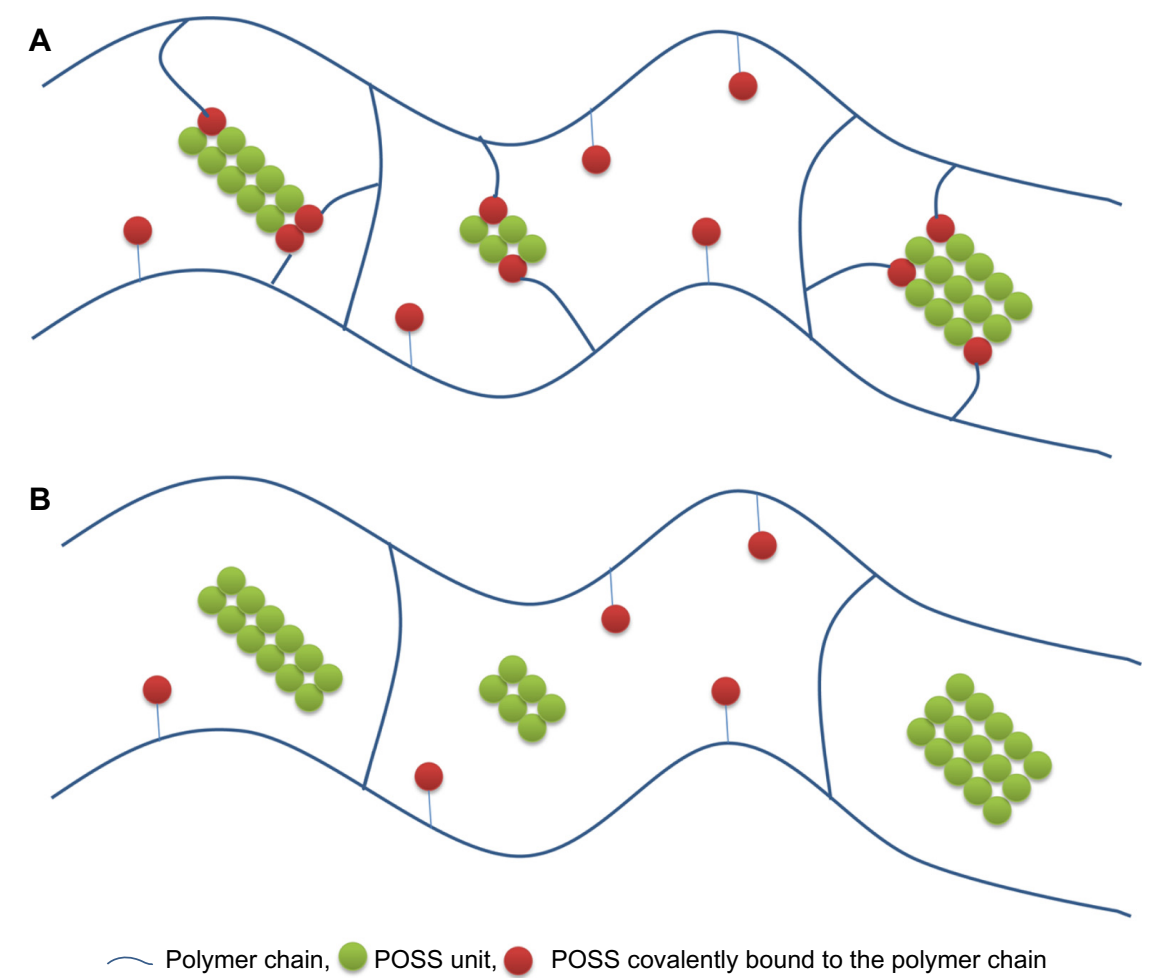

Figure 5 POSS distribution in the polymer matrix shown as (A) polymer matrix with MA-POSS and (B) polymer matrix with MI-POSS

Abbreviations: MA-POSS, methacryl polyhedral oligomeric silsesquioxanes; MI-POSS, methacryl isobutyl polyhedral oligomeric silsesquioxanes; POSS, polyhedral oligomeric silsesquioxanes. 

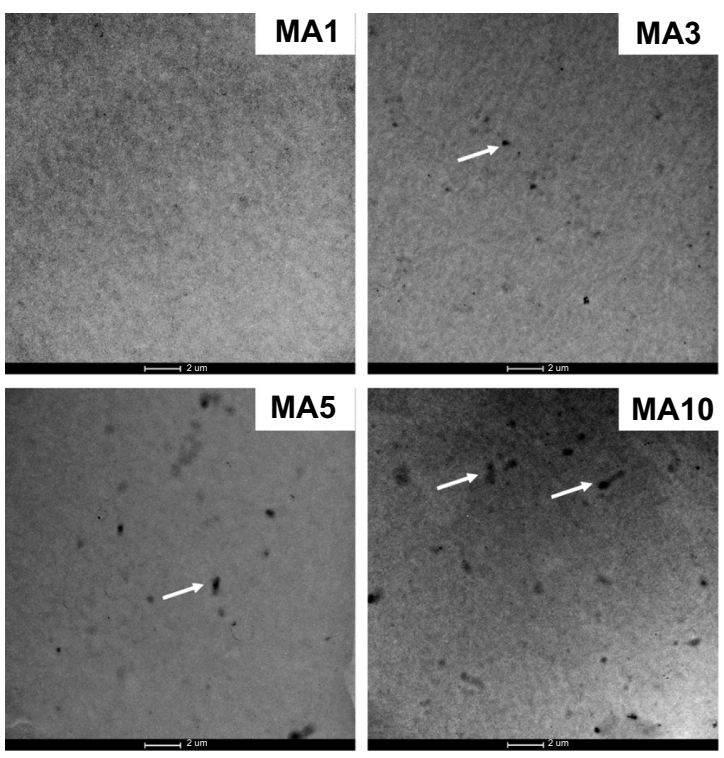

Figure 6 Transmission electron microscope micrographs of resins filled with MA-POSS.

Notes: (MAI) Resin with I wt\% of methacryl polyhedral oligomeric silsesquioxanes group. (MA3) Resin with 3 wt\% of methacryl polyhedral oligomeric silsesquioxanes. (MA5) Resin with 5 wt\% of methacryl polyhedral oligomeric silsesquioxanes. (MA 10) Resin with 10 wt\% of methacryl polyhedral oligomeric silsesquioxanes. Arrows show the aggregation of MA-POSS.

Abbreviation: MA-POSS, methacryl polyhedral oligomeric silsesquioxanes.

morphological structures of large agglomerates, but it is difficult to resolve small agglomerates from the matrix because of the complexity of the polymer microstructure.

\section{Degree of conversion}

In conventional dental composite materials, DC is highly correlated with the mechanical and shrinkage properties of the material, ${ }^{22,23}$ which may be explained by the higher density achieved in the densely compacted cross-network of dental composite resins. In this study, DC was studied to determine whether the Bis-GMA/TEGDMA dental resins filled by POSS could be appropriately photocured.

Figure 7 shows the representative FTIR spectra of the control, MA10, and MI10 groups in the region of $1,660-1,590 \mathrm{~cm}^{-1}$. Bands at $1,637 \mathrm{~cm}^{-1}$ represent the aliphatic stretching vibrations of the resin matrix; in these materials it is mostly influenced by the Bis-GMA, TEGDMA, and POSS. Aromatic bands at $1,607 \mathrm{~cm}^{-1}$ were taken as an internal standard. As can be seen by comparing the spectra of cured and uncured material samples, the absorbance of the aliphatic band is reduced after polymerization. The average $\mathrm{DC} \%$ of different POSS types and concentrations filled with neat resin at different time points from the start of irradiation are displayed graphically in Figure 8. The degree of conversion increased dramatically during the first 60 seconds and con- tinued to rise until a plateau was reached at approximately 100 seconds. The final conversion of double bonds decreases with the increasing MA-POSS and MI-POSS content, and the latter produces a comparatively greater impact. This may be explained by the steric hindrance and the reduction of polymer network mobility associated with the inorganic part of these POSS. In addition, the agglomerations generated by POSS (especially MI-POSS) may result in limited chemical reaction of the organic part of POSS.

\section{WAXD}

The WAXD patterns of $1,3,5$, and $10 \mathrm{wt} \%$ MA-POSS and MI-POSS resins are shown in Figure 9, together with those of the pure MI-POSS powder and the control resin matrix (without POSS) to serve as references. The WAXD data (Figure 9) indicated that the control resin matrix exhibited diffuse amorphous halos at $2 \theta=18.7^{\circ}$. Pure MI-POSS exhibits characteristic crystalline peaks at $2 \theta=7.3^{\circ}, 8.0^{\circ}, 10.0^{\circ}, 11.76^{\circ}, 18.8^{\circ}$, and $19.6^{\circ}$. The presence of sharp peaks in the WAXD patterns of MI-POSS samples indicated their highly crystalline nature. All MAPOSS nanocomposites spectra appeared very similar to that of the resin matrix, with no evident crystalline peaks, even when the mass fraction of the MA-POSS monomers in the resin mixture was as high as $10 \%$. The WAXD spectrum of MI1, MI3, MI5, and MI10, on the other hand, showed the amorphous halo of control resin matrix and crystalline peaks corresponding to neat MI-POSS. These crystalline peaks indicated that POSS could aggregate in the matrix as microcrystals. As POSS loading increased, the intensity of this crystalline peak in the composite increased. Thus, more and larger particles existed, which is consistent with the SEM results.

\section{Volumetric shrinkage}

During the polymerization of methacrylate-based resins, the viscous liquid monomers gradually transform into a rigid material by radical polymerization involving the $\mathrm{C}=\mathrm{C}$ double bonds of methacrylate groups. When monomers in proximity react to establish a covalent bond, the distance between the two groups of atoms is reduced and there is a reduction in free volume, both of which translate into volumetric shrinkage. Contemporary commercial dental composites have polymerization shrinkage ranging from $2 \%-5.63 \%{ }^{24}$ This polymerization shrinkage creates contraction stresses in the composite resin restoration and internal stress and deformation in the surrounding tooth structure. ${ }^{24}$ As a consequence, marginal leakage of saliva and its components 

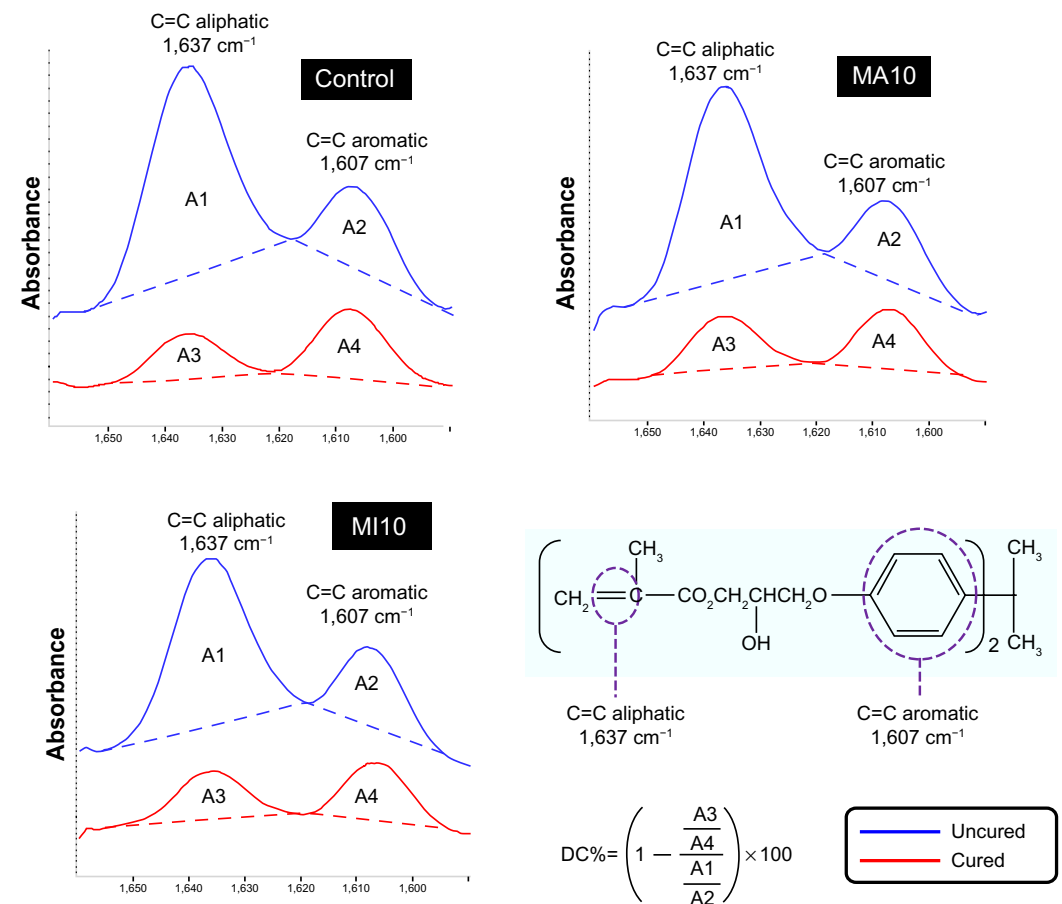

Figure 7 Fourier transform infrared spectrometry spectra of an uncured and cured specimen for control, MAI0, MIIO showing the absorbance peaks of the aliphatic $\mathrm{C}=\mathrm{C}$ bond $\left(\mathrm{I}, 637 \mathrm{~cm}^{-1}\right)$ and the aromatic $\mathrm{C}=\mathrm{C}$ bond $\left(\mathrm{I}, 607 \mathrm{~cm}^{-1}\right)$ used to calculate the $\mathrm{DC} \%$.

Abbreviations: DC\%, degree of double bond conversion; MAI0, resin with $10 \mathrm{wt} \%$ of methacryl polyhedral oligomeric silsesquioxanes group; MII0, resin with $10 \mathrm{wt} \%$ of methacryl isobutyl polyhedral oligomeric silsesquioxanes group.
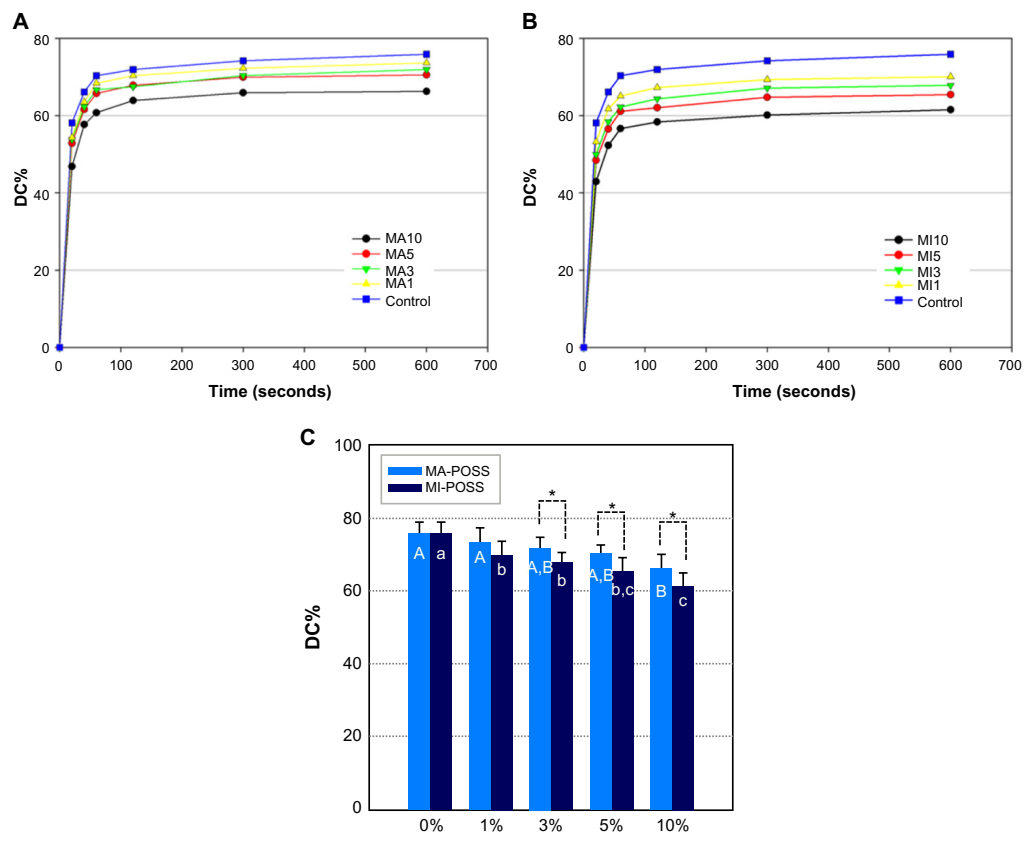

Poss-concentration

Figure 8 The average degree of double bond conversion of different POSS types and concentrations filled with neat resin at different time points from the start of irradiation.

Notes: The DC\% using MA-POSS (A), MI-POSS (B), and resin filled with varied mass fractions of MA-POSS and MI-POSS (C). Data are presented as mean and standard deviation in $\mathbf{C}, \mathrm{n}=5$, two-way analysis of variance. Uppercase letters represent the statistical result of MA-POSS under different concentrations, and lowercase letters represent the statistical result of MI-POSS under different concentrations. If the same letters aren't shown on the two bars, the result of the two concentrations was found to be statistically different. *Indicates significant differences between two types of POSS $(P<0.05)$

Abbreviations: DC\%, degree of double bond conversion; MA-POSS, methacryl polyhedral oligomeric silsesquioxanes; MI-POSS, methacryl isobutyl polyhedral oligomeric silsesquioxanes; POSS, polyhedral oligomeric silsesquioxanes; MAI, resin with I wt\% of methacryl polyhedral oligomeric silsesquioxanes group; MA3, resin with 3 wt\% of methacryl polyhedral oligomeric silsesquioxanes; MA5, resin with $5 \mathrm{wt} \%$ of methacryl polyhedral oligomeric silsesquioxanes; MA I0, resin with I0 wt\% of methacryl polyhedral oligomeric silsesquioxanes; MII, resin with I wt\% of methacryl isobutyl polyhedral oligomeric silsesquioxanes group; MI3, resin with 3 wt\% of methacryl isobutyl polyhedral oligomeric silsesquioxanes; MI5, resin with $5 \mathrm{wt} \%$ of methacryl isobutyl polyhedral oligomeric silsesquioxanes; MII0, resin with 10 wt\% of methacryl isobutyl polyhedral oligomeric silsesquioxanes. 


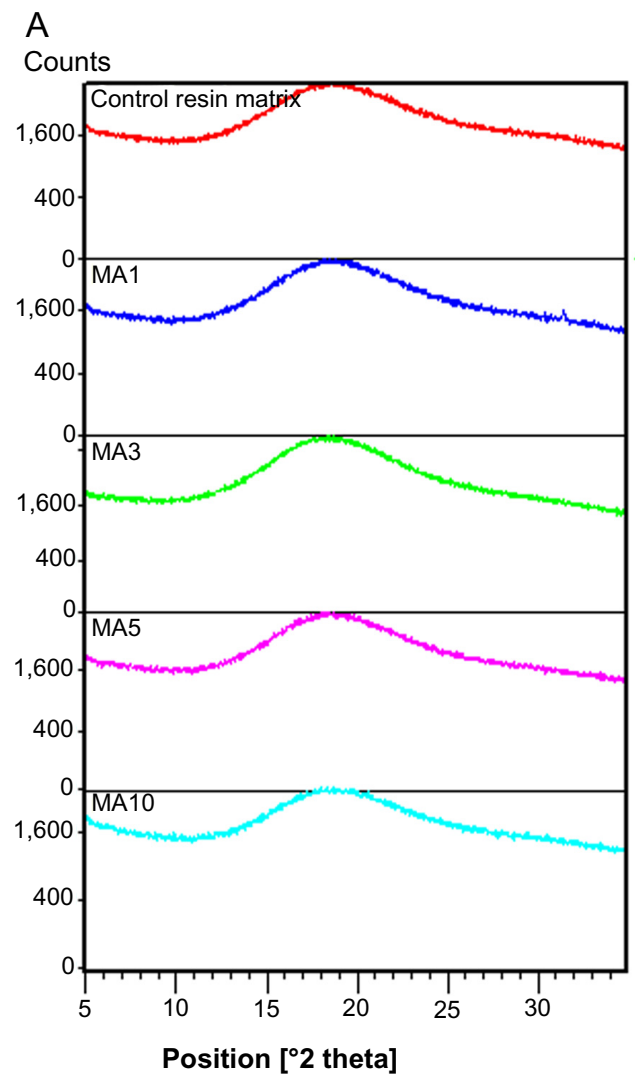

\section{B}

Counts

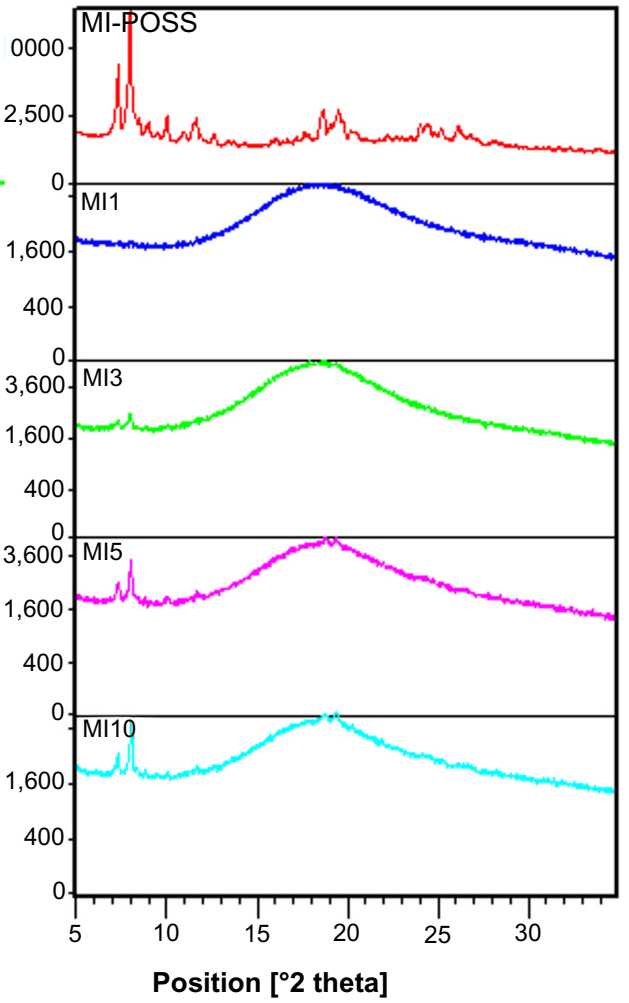

Figure 9 Wide-angle X-ray diffraction curves of the resin samples and pure MI-POSS, with (A) neat resin matrix and resins filled with MA-POSS and (B) pure MI-POSS and resins filled with MI-POSS.

Notes: (MAI) Resin with I wt\% of methacryl polyhedral oligomeric silsesquioxanes group. (MA3) Resin with 3 wt\% of methacryl polyhedral oligomeric silsesquioxanes. (MA5) Resin with $5 \mathrm{wt} \%$ of methacryl polyhedral oligomeric silsesquioxanes. (MAI0) Resin with $10 \mathrm{wt} \%$ of methacryl polyhedral oligomeric silsesquioxanes. (MII) Resin with I wt\% of methacryl isobutyl polyhedral oligomeric silsesquioxanes group. (MI3) Resin with 3 wt\% of methacryl isobutyl polyhedral oligomeric silsesquioxanes. (MI5) Resin with 5 wt\% of methacryl isobutyl polyhedral oligomeric silsesquioxanes. (MII 0) Resin with 10 wt\% of methacryl isobutyl polyhedral oligomeric silsesquioxanes. Abbreviations: MA-POSS, methacryl polyhedral oligomeric silsesquioxanes; MI-POSS, methacryl isobutyl polyhedral oligomeric silsesquioxanes.

will occur, resulting in postoperative sensitivity, discolored margins, recurrent caries, and fractures of the restoration margins. ${ }^{25}$ These clinical consequences are the main reasons for premature replacement composite of resin restorations and explain why polymerization shrinkage is recognized as the main limitation of dental composite resins. ${ }^{26}$

In this study, the volumetric polymerization shrinkages of filled composite resins (70\% filler) are summarized in Figure 10. For both MA-POSS-filled and MI-POSS-filled resins, shrinkage decreased as the volume of addition increased. The results proved that both multifunctional and monofunctional methacryl POSS can effectively reduce the shrinkage of composite resins and that shrinkage is reduced with increasing percentage of POSS. The volumetric shrinkage is closely related to the methacrylate $\mathrm{DC}$, which is a major factor influencing the properties of the cured composites. ${ }^{27}$ The decrease in DC (Figure 8) of MA-POSS and MI-POSS may be the most important reason for the decrease in shrinkage. It remains a technological challenge for dental composites to possess both low volumetric shrinkage and high DC at the same time. ${ }^{1}$ In addition, it is possible that the special nanocubic structure of POSS limits the change of the resins' free volume after a cross-linked net framework is formed. ${ }^{15}$

\section{Mechanical properties}

FS and $\mathrm{E}$ of Bis-GMA/TEGDMA dental composites containing various mass fractions of MA-POSS and MI-POSS were tested, and the results are shown in Figure 11. The control sample was the composite without POSS. Each datum in the plots represents the mean value of six measurements with standard deviation. As shown in Figure 11, adding MI-POSS to the composite significantly decreased its FS and E values, probably because MI-POSS agglomerates easily, and the size of the agglomerates often reaches $20 \mu \mathrm{m}$. If more MI-POSS is added to the material, then more and larger agglomerates will appear in the composites. Such large agglomerates are detrimental to the mechanical properties of the composite.

In contrast to MI-POSS, the FS and E values were increased by the impregnation of small amounts of MA-POSS into the dental composite resins. The FS was improved by $30.2 \%$ with 


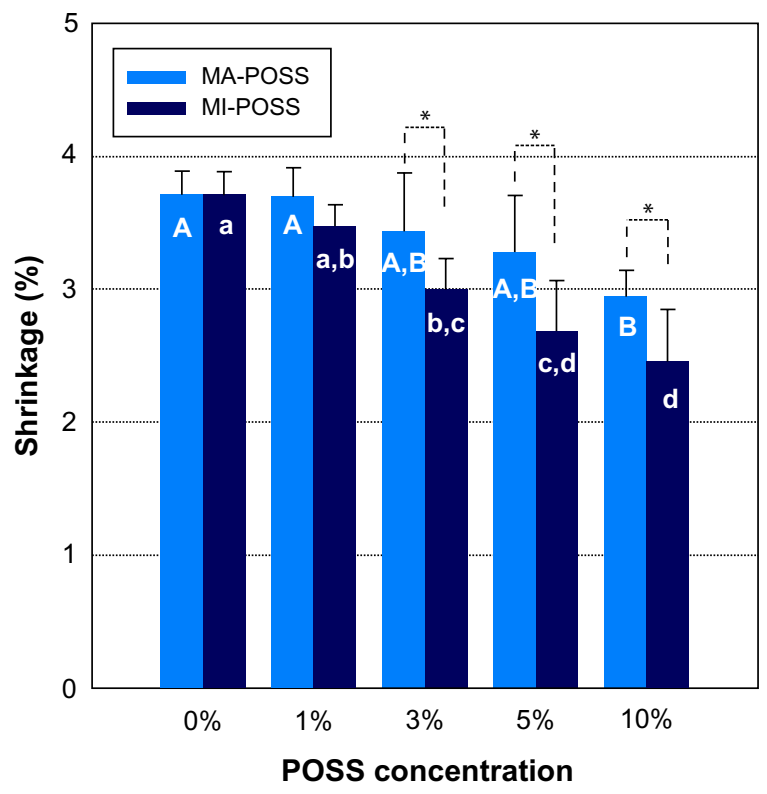

Figure 10 The shrinkage of Bis-GMA/TEGDMA dental composites filled with varied mass fractions of MA-POSS and MI-POSS.

Notes: Data are presented as mean and standard deviation, $n=6$, two-way analysis of variance. Uppercase letters represent the statistical result of MA-POSS under different concentrations, and lowercase letters represent the statistical result of MI-POSS under different concentrations. If the same letters aren't shown on the two bars, the result of the two concentrations was found to be statistically different. *Indicates significant differences between two types of POSS $(P<0.05)$.

Abbreviations: Bis-GMA, bisphenol A glycerolate dimethacrylate; TEGDMA, tri(ethylene glycol) dimethacrylate; MA-POSS, methacryl polyhedral oligomeric silsesquioxanes; MI-POSS, methacryl isobutyl polyhedral oligomeric silsesquioxanes.

$3 \mathrm{wt} \%$ of MA-POSS and E was improved by $22.3 \%$ with $5 \mathrm{wt} \%$ of MA-POSS. However, further increasing the mass fractions of the MA-POSS did not increase the mechanical properties. On the contrary, the FS and E values decreased

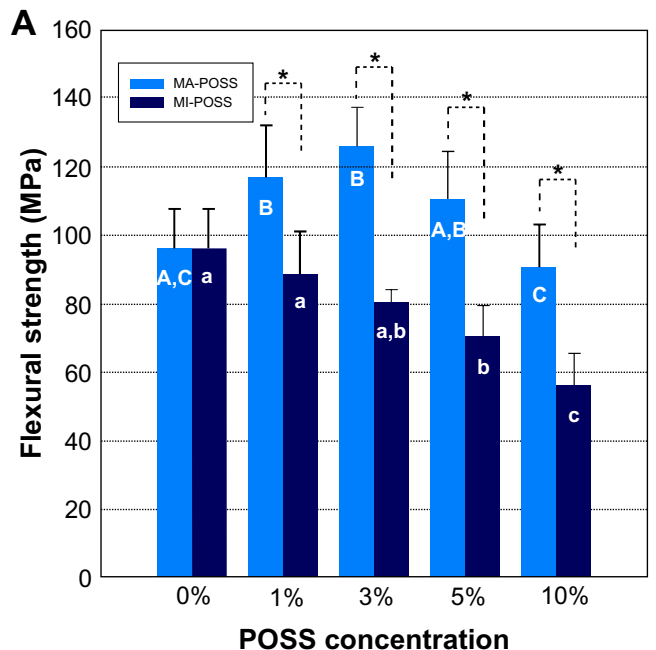

with larger proportions of MA-POSS. The impregnation of the MA-POSS into Bis-GMA/TEGDMA dental composite resins may have a double-edged effect: a reinforcing effect caused by the well-dispersed MA-POSS and a weakening effect caused by the formation of MA-POSS agglomerates. The well-dispersed POSS moiety served as a junction point in the network architecture. It reinforced the networks by increasing the density of cross-linking and constraining the molecular motion in the rigid region around the inorganic POSS unit. When the MA-POSS mass fraction was less than $5 \mathrm{wt} \%$, a small portion of MA-POSS form agglomerates, and most of the MA-POSS is probably well-distributed in the resin matrix (Figure 6). When the MA-POSS mass fraction increased to $10 \mathrm{wt} \%$, more agglomerates were formed in the dental resin matrix (Figures 2E and 6), which can act as the mechanical weak point in the dental resins and lead to the observed lower $\mathrm{FS}$ and $\mathrm{E}$ value at the $10 \mathrm{wt} \%$ mass fraction.

MA-POSS has eight methacrylate functional groups. In the process of polymerization, not all the methacrylate functional groups can react into the polymer chain due to steric hindrance associated with the inorganic part of POSS. In addition, some MA-POSS form aggregates. These unreacted methacrylate functional groups in MA-POSS may result in a decrease of DC. However, MA-POSS may work as a stabilizer or cross-linker in the resin matrix, as long as there are more than three methacrylate functional groups in the POSS molecules reacted into polymer chains. Thus, when the MA-POSS concentration is low, the partly reacted MA-POSS is the main cause of decreased DC. The

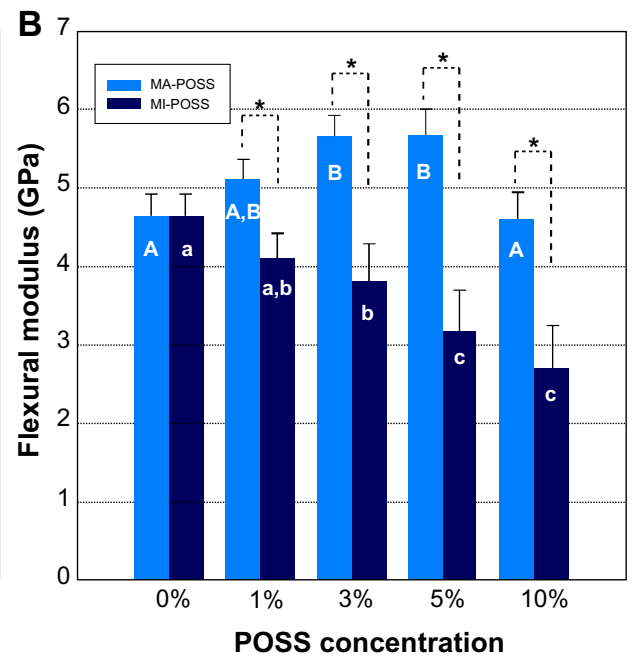

Figure II The flexural strength (A), flexural modulus (B) of Bis-GMA/TEGDMA dental composites filled with varied mass fractions of MA-POSS and MI-POSS. Notes: Data are presented as mean and standard deviation, $n=6$, two-way analysis of variance. The same letters in the bar indicate no significant differences of different POSS concentration ( $P>0.05)$; Uppercase letters represent the statistical result of MA-POSS under different concentrations, and lowercase letters represent the statistical result of MI-POSS under different concentrations. If the same letters aren't shown on the two bars, the result of the two concentrations was found to be statistically different.*Indicates significant differences between two types of POSS $(P<0.05)$.

Abbreviations: Bis-GMA, bisphenol A glycerolate dimethacrylate; MA-POSS, methacryl polyhedral oligomeric silsesquioxanes; MI-POSS, methacryl isobutyl polyhedral oligomeric silsesquioxanes; TEGDMA, tri(ethylene glycol) dimethacrylate. 
A

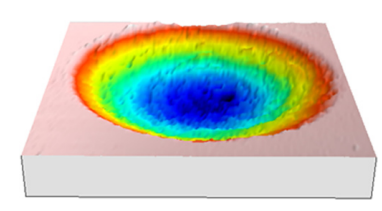

C

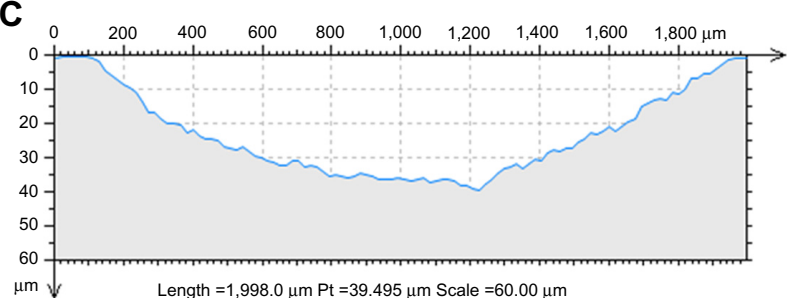

B

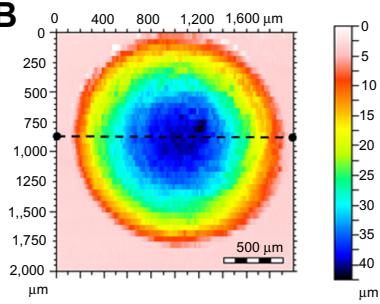

Length $=1,998.0 \mu \mathrm{m} \mathrm{Pt}=39.495 \mu \mathrm{m}$ Scale $=60.00 \mu \mathrm{m}$

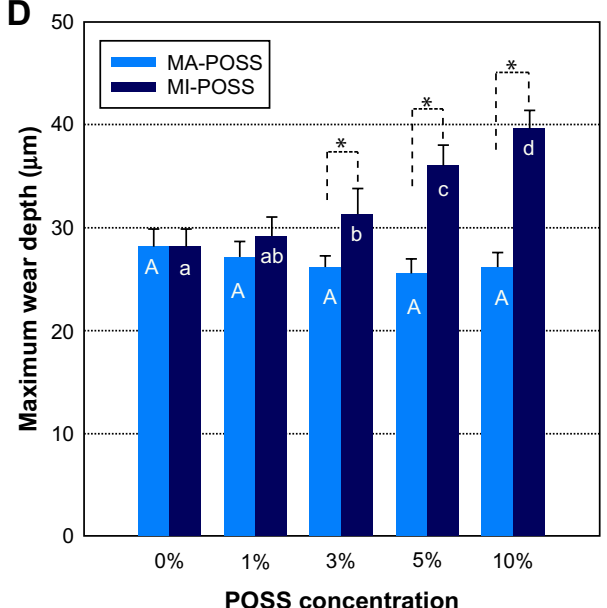

Figure 12 Test methods and results of the maximum wear depth tests.

Notes: (A) Three-dimensional representation of a wear facet. (B) Two-dimensional representation of the wear facet. (C) Extracted vertical profile of B. (D) The maximum wear depth of dental composites filled with varied mass fractions of MA-POSS and MI-POSS.

Notes: Data are presented as mean and standard deviation, $n=6$, two-way analysis of variance. The same letters in the bar indicate no significant differences of different POSS concentration $(P>0.05)$. Uppercase letters represent the statistical result of MA-POSS under different concentrations, and lowercase letters represent the statistical result of MI-POSS under different concentrations. If the same letters aren't shown on the two bars, the result of the two concentrations was found to be statistically different. *Indicates significant differences between two types of POSS $(P<0.05)$.

Abbreviations: MA-POSS, methacryl polyhedral oligomeric silsesquioxanes; MI-POSS, methacryl isobutyl polyhedral oligomeric silsesquioxanes.

partial or complete reacted MA-POSS works as a stabilizer or cross-linker in the resin matrix, which is the main cause of increased strength. When the MA-POSS concentration is high, the aggregation of MA-POSS is the main cause of the decrease in DC and strength.

According to ISO 4049-2009, the threshold for the FS of dental resin composites is $80 \mathrm{Mpa}$, while contemporary commercial dental composites have flexural strengths typically ranging from $99-142 \mathrm{MPa} .{ }^{28,29}$ The flexural strengths of the experimental composites reinforced by $3 \mathrm{wt} \%$ MA-POSS have higher flexural strengths than that of many commercial composites. A good selection of filler and optimization of its content in future research may further enhance the strength of the composite.

\section{Wear resistance}

Wear is the loss of material that occurs through contact of two or more surfaces. In dental composites, wear involves the process of resin matrix loss between filler particles and subsequent dislodgement of the filler. ${ }^{30}$ High resistance to wear is one of the desirable properties for restorative materials. Bhamra and Fleming reported that the maximum wear depth of commercial composites is about 40-90 $\mu \mathrm{m}$ in 50,000 cycles of wear. ${ }^{31}$ As shown in Figure 12, the authors used a noncontact profilometer to scan the worn zone, and then extracted the vertical profile to detect the maximum wear depth. The results of maximum wear depth and standard deviations of composite resin are presented in Figure 12D. The maximum wear depth of the MA-POSS composites showed a tendency to decrease with increasing MA-POSS concentration but was not statistically significant $(P>0.05)$. By contrast, the values of maximum wear depth increased with increasing amounts of MI-POSS in the dental composites. There were significant increases in the maximum wear depth data of MI3, MI5, and MI10 compared with the control group. The low wear resistance of the MI-POSS composites may be related to the relatively low degree of conversion and the mechanical properties of the composites.

\section{Conclusion}

We have demonstrated that the properties of the two studied POSS-containing hybrids are closely related to their structure and morphology. The mechanical properties are worse in the case of the hybrid network with pendant monofunctional MIPOSS, which forms nonhomogeneously dispersed agglomerates in the dental matrices. However, the multifunctional POSS monomer (MA-POSS) is homogeneously dispersed in the methacrylate network as a polyhedral junction at low POSS concentrations. The homogeneous nanocomposite reinforced by $3 \mathrm{wt} \%$ MA-POSS exhibits significantly improved mechanical and shrinkage properties. Thus, dental nanocomposites based on $3 \mathrm{wt} \%$ MA-POSS represent promising materials for dental applications. 


\section{Acknowledgments}

This work was supported by grants from the National Natural Science Foundation of China (No 81371187, No 81130078, and No 81200816). We thank Professor Hong Wu, School of Pharmacy, Fourth Military Medical University, for her help and for allowing this work to progress in her laboratory.

\section{Disclosure}

The authors report no conflicts of interest in this work.

\section{References}

1. Chen Q, Zhao Y, Wu W, Xu T, Fong H. Fabrication and evaluation of Bis-GMA/TEGDMA dental resins/composites containing halloysite nanotubes. Dent Mater. 2012;28(10):1071-1079.

2. Stein PS, Sullivan J, Haubenreich JE, Osborne PB. Composite resin in medicine and dentistry. $J$ Long Term Eff Med Implants. 2005;15(6):641-654.

3. Chen L, Yu Q, Wang Y, Li H. BisGMA/TEGDMA dental composite containing high aspect-ratio hydroxyapatite nanofibers. Dent Mater. 2011;27(11):1187-1195.

4. Liu WW, He XP, Mo AC, Yao QQ, Ye J, Jing N. Investigation of the mechanical properties of a low-shrinkage liquid crystalline matrix combined with nano-hydroxyapatite. Int J Nanomedicine. 2011;6:1787-1791.

5. Lin S, Cai Q, Ji J, et al. Electrospun nanofiber reinforced and toughened composites through in situ nano-interface formation. Composites $S_{c i}$ Technol. 2008;68(15-16):3322-3329.

6. Sarrett DC. Clinical challenges and the relevance of materials testing for posterior composite restorations. Dent Mater. 2005;21(1):9-20.

7. Cho H, Liang K, Chatterjee S, Pittman CU Jr. Synthesis, morphology, and viscoelastic properties of polyhedral oligomeric silsesquioxane nanocomposites with epoxy and cyanate ester matrices. J Inorg Organomet Polym Mater. 2005;15(4):541-553.

8. Rizvi SB, Yildirimer L, Ghaderi S, Ramesh B, Seifalian AM, Keshtgar M. A novel POSS-coated quantum dot for biological application. Int $J$ Nanomedicine. 2012;7:3915-3927.

9. Ghanbari H, de Mel A, Seifalian AM. Cardiovascular application of polyhedral oligomeric silsesquioxane nanomaterials: a glimpse into prospective horizons. Int J Nanomedicine. 2011;6:775-786.

10. Chen P, Huang X, Zhang Q, Xi K, Jia X. Hybrid networks based on poly (styrene-co-maleic anhydride) and N-phenylaminomethyl POSS. Polymer. 2013;54(3):1091-1097.

11. Li G, Wang L, Ni H, Pittman CU Jr. Polyhedral oligomeric silsesquioxane (POSS) polymers and copolymers: a review. J Inorg Organomet Polym. 2001;11(3):123-154.

12. Frank KL, Exley SE, Thornell TL, Morgan SE, Wiggins JS. Investigation of pre-reaction and cure temperature on multiscale dispersion in POSS-epoxy nanocomposites. Polymer. 2012;53(21):4643-4651.

13. Matějka L, Strachota A, Pleštil J, Whelan P, Steinhart M, Šlouf M. Epoxy networks reinforced with polyhedral oligomeric silsesquioxanes (POSS). Structure and morphology. Macromolecules. 2004;37(25):9449-9456.

International Journal of Nanomedicine

\section{Publish your work in this journal}

The International Journal of Nanomedicine is an international, peerreviewed journal focusing on the application of nanotechnology in diagnostics, therapeutics, and drug delivery systems throughout the biomedical field. This journal is indexed on PubMed Central, MedLine, CAS, SciSearch $\AA$, Current Contents $₫ /$ Clinical Medicine,
14. Zhao Y, Schiraldi DA. Thermal and mechanical properties of polyhedral oligomeric silsesquioxane (POSS)/polycarbonate composites. Polymer. 2005;46(25):11640-11647.

15. Wu X, Sun Y, Xie W, Liu Y, Song X. Development of novel dental nanocomposites reinforced with polyhedral oligomeric silsesquioxane (POSS). Dent Mater. 2010;26(5):456-462.

16. Soh MS, Yap AUJ, Sellinger A. Methacrylate and epoxy functionalized nanocomposites based on silsesquioxane cores for use in dental applications. Eur Polym J. 2007;43(2):315-327.

17. Fong H, Dickens SH, Flaim GM. Evaluation of dental restorative composites containing polyhedral oligomeric silsesquioxane methacrylate. Dent Mater. 2005;21(6):520-529.

18. Yen YC, Ye YS, Cheng CC, et al. The effect of sulfonic acid groups within a polyhedral oligomeric silsesquioxane containing cross-linked proton exchange membrane. Polymer. 2010;51(1):84-91.

19. Boček J, Matějka L, Mentlík V, Trnka P, Šlouf M. Electrical and thermomechanical properties of epoxy-POSS nanocomposites. Eur Polym J. 2011;47(5):861-872.

20. Liu H, Zheng S, Nie K. Morphology and thermomechanical properties of organic-inorganic hybrid composites involving epoxy resin and an incompletely condensed polyhedral oligomeric silsesquioxane. Macromolecules. 2005;38(12):5088-5097.

21. Liang K, Toghiani H, Pittman CU Jr. Synthesis, morphology and viscoelastic properties of epoxy/polyhedral oligomeric silsesquioxane (POSS) and epoxy/cyanate ester/POSS nanocomposites. J Inorg Organomet Polym. 2011;21(1):128-142.

22. Kuşgöz A, Tüzüner T, Ulker M, Kemer B, Saray O. Conversion degree, microhardness, microleakage and fluoride release of different fissure sealants. J Mech Behav Biomed Mater. 2010;3(8):594-599.

23. Marovic D, Panduric V, Tarle Z, et al. Degree of conversion and microhardness of dental composite resin materials. J Mol Struct. 2013;1044(24):299-302.

24. Kleverlaan CJ, Feilzer AJ. Polymerization shrinkage and contraction stress of dental resin composites. Dent Mater. 2005;21(12):1150-1157.

25. Petrovic LM, Atanackovic TM. A model for shrinkage strain in photo polymerization of dental composites. Dent Mater. 2008;24(4):556-560.

26. Dewaele M, Truffier-Boutry D, Devaux J, Leloup G. Volume contraction in photocured dental resins: the shrinkage-conversion relationship revisited. Dent Mater. 2006;22(4):359-365.

27. Ferracane JL, Greener EH. The effect of resin formulation on the degree of conversion and mechanical properties of dental restorative resins. J Biomed Mater Res. 1986;20(1):121-131.

28. Sideridou ID, Karabela MM, Vouvoudi ECh. Physical properties of current dental nanohybrid and nanofill light-cured resin composites. Dent Mater. 2011;27(6):598-607.

29. Monteiro GQ, Montes MA. Evaluation of linear polymerization shrinkage, flexural strength and modulus of elasticity of dental composites. Mat Res. 2010;13(1):51-55.

30. Hammouda IM. Effect of light-curing method on wear and hardness of composite resin. J Mech Behav Biomed Mater. 2010;3(2):216-222.

31. Bhamra GS, Fleming GJ. Influence of halogen irradiance on short- and long-term wear resistance of resin-based composite materials. Dent Mater. 2009;25(2):214-220.

Journal Citation Reports/Science Edition, EMBase, Scopus and the Elsevier Bibliographic databases. The manuscript management system is completely online and includes a very quick and fair peer-review system, which is all easy to use. Visit http://www.dovepress.com/ testimonials.php to read real quotes from published authors. 